\title{
PRODUCCIÓN CIENTÍFICA Y EVOLUCIÓN CONCEPTUAL DEL MERCHANDISING DURANTE LAS ÚLTIMAS SEIS DÉCADAS. UN ESTUDIO BIBLIOMÉTRICO
}

\section{SCIENTIFIC PRODUCTION AND CONCEPTUAL EVOLUTION OF SELF-SERVICE MERCHANDISING DURING THE LAST SIX DECADES. A BIBLIOMETRIC STUDY}

Francisco Muñoz Leiva (Universidad de Granada) ${ }^{1}$

$\mathbf{M}^{\mathbf{a}}$. Eugenia Rodríguez López (Universidad de Granada) ${ }^{2}$

Francisco Liébana Cabanillas (Universidad de Granada) ${ }^{3}$

\section{Resumen:}

En este artículo aparecen los resultados de un análisis bibliométrico centrado en la comunidad académica y profesional interesada por el estudio del merchandising de autoservicio (selfservice merchandising) o marketing en el punto de venta. El objetivo es identificar los temas pasados y actuales, predecir las tendencias emergentes y proporcionar una perspectiva longitudinal sobre la investigación que ha tenido lugar entre 1960 y 2019, a partir de unos 700 artículos extraídos de la base bibliográfica Scopus. Por tanto, este enfoque proporciona el análisis más exhaustivo de la historia del merchandising en las últimas seis décadas.

Algunos de los hallazgos muestran que la década pasada se caracterizó por el estudio de simuladores como CAD, y de la gestión de la cadena de suministro aplicado al sector textil. Si bien, el merchandising visual no es algo nuevo, se trata una temática emergente en este campo de investigación que irá integrando el papel del merchandising virtual, la atmósfera del establecimiento y la aplicación de técnicas de escaparatismo. En esta década, la publicidad aparece como un tema general o transversales a otras temáticas. Y el futuro pasará por la aplicación de la realidad virtual y la realidad aumentada al caso del e-merchandising.

Las conclusiones y futuras líneas de investigación contribuyen a avanzar en el conocimiento y la comprensión de la literatura académica sobre el merchandising o el marketing en el punto de venta.

Palabras clave: Estudio bibliométrico, análisis co-word, merchandising, marketing en el punto de venta.

Códigos JEL: M30, M31.

\footnotetext{
1 franml@ugr.es, Facultad de Ciencias Económicas y Empresariales de la Universidad de Granada. Campus Universitario La Cartuja, s/n. 18071 Granada (España).

2 eugeniarodriguez@ugr.es, Facultad de Educación, Economía y Tecnología de Ceuta. Cortadura del Valle, s/n, 51001 Ceuta.

3 franlieb@ugr.es, Facultad de Ciencias Económicas y Empresariales de la Universidad de Granada. Campus Universitario La Cartuja, s/n. 18071 Granada.

Recibido: 1 de abril de 2020. Aceptado: 9 de junio de 2020.
} 


\begin{abstract}
:
This article shows the results of a bibliometric analysis focused on the academic and professional community interested in the study of self-service merchandising or marketing at the point of sale. The objective is to identify past and current issues, predict emerging trends and provide a longitudinal perspective on the research that took place between 1960 and 2019, from around 700 articles taken from the Scopus bibliographic database. Therefore, this approach provides the most comprehensive analysis of the history of point-of-sale marketing in the past six decades.

Some of the findings found in the past decade are characterized by the study of simulators such as CAD, and supply chain management applied to the textile sector. Although visual merchandising is not new, it is an emerging theme in this field of research that will gradually integrate the role of virtual merchandising, the atmosphere of the establishment and the application of window dressing techniques. In this decade, advertising appear as a general or transversal topic to others topics. And the future will go through the application of virtual reality and augmented reality to the case of e-merchandising.

Conclusions and future lines of research advanced to advance knowledge and understanding of the academic literature on marketing or point-of-sale marketing.
\end{abstract}

Keywords: Bibliometric study, co-word analysis, merchandising, point-of-sale marketing.

JEL codes: M30, M31.

\title{
1. INTRODUCCIÓN
}

Con el régimen de libre servicio en el ámbito comercial, la evolución de diversas técnicas de marketing empleadas en el punto de venta ha derivado en diversos tipos de merchandising: por un lado, el merchandising de presentación de producto y el estratégico o de gestión (Palomares, 2011), y por otro merchandising de seducción y el de fidelización (Martínez, 2005). Sin embargo, y a pesar del peso que el merchandising demuestra tener a lo largo de la literatura académica no se observa hasta la fecha ningún estudio bibliométrico que aborde su desarrollo y evolución temática.

Para avanzar en el conocimiento de cualquier disciplina científica, es necesario atender a las contribuciones teóricas y empíricas de los académicos y profesionales a lo largo del tiempo, por lo que un análisis periódico del conocimiento acumulado es necesario para comprender el estado actual de una disciplina (Rodríguez-López et al., 2019).

Este trabajo trata de superar este gap identificado para aportar un mayor conocimiento del estado de la cuestión en las técnicas de merchandising o marketing en el punto de venta, centrándose en el periodo 1961-2019, para un análisis exhaustivo, aplicando una orientación bibliométrica mediante análisis de co-ocurrencia de palabras y mapas de ciencia.

El análisis bibliométrico es frecuentemente utilizado para conocer este estado del arte de un área de conocimiento, ya que proporciona información cuantitativa para evaluar la investigación de una disciplina específica. Esta información está basada en el análisis de palabras clave, su co-ocurrencia, el número de citas, el año de publicación, los autores más prolíficos, su afiliación, fuentes más citadas o que más publican sobre un tema, así como otras informaciones disponibles en las bases de datos bibliográficas. En contraposición, las revisiones cualitativas de literatura académica proporcionan una imagen profunda de un tema a partir de 
la identificación de diferentes enfoques a lo largo del tiempo y de las relaciones que existen entre ellos (Muñoz-Leiva et al., 2015).

Son diversos los autores que han utilizado diferentes análisis bibliométricos para resumir el conocimiento científico en diferentes disciplinas como los negocios o el marketing, incluyendo la investigación en sistemas de gestión de información (Culnan, 1986), la gestión estratégica (Ramos-Rodríguez y Ruiz-Navarro, 2004; Vogel y Güttel, 2013), el marketing financiero (Muñoz et al., 2012a; 2013), la comunicación integrada de marketing (Muñoz et al., 2015) o comportamiento del consumidor (Muñoz-Leiva et al., 2012b).

En particular, el análisis de co-ocurrencia de palabras clave (“co-word analysis”) es una técnica bibliométrica que proporciona información de los intereses de los investigadores en un campo específico de investigación (Cornelius y Persson, 2006). Esta técnica permite identificar, describir y visualizar las interacciones entre palabras clave, términos y temas, y es aplicable en cualquier ámbito de investigación científica (ej. Callon et al., 1991; Bailón-Moreno et al., 2006; Muñoz-Leiva et al., 2012a; 2012b; 2013; 2015). Más específicamente, al analizar la frecuencia de co-ocurrencia de palabras se está contabilizando el número de documentos en los cuales dos palabras aparecen juntas. En este contexto, los mapas de la ciencia o mapas científicos derivados tienen por objeto mostrar los aspectos estructurales y dinámicos de la investigación científica (ej. Börner et al., 2003; Morris y Van Der Veer Martens, 2008).

En el presente trabajo se aborda a continuación el origen del merchandising como técnica comercial, seguido de una descripción de los tipos de merchandising más relevantes en los que el término ha evolucionado. En tercer lugar, se describe exhaustivamente el procedimiento de análisis co-word llevado a cabo, y finalmente se presentan los resultados del análisis de rendimiento y de contenido aplicado. La materia prima usada para este análisis está compuesta por todos los artículos publicados sobre merchandising e indexados en la base bibliográfica Scopus. Con ello se pretende describir el progreso de este campo de investigación, conocer los temas que han centrado el interés e identificar las tendencias emergentes y por tanto hacia dónde se dirigirán las investigaciones futuras.

\section{CONCEPTUALIZACIÓN Y TIPOS DE MERCHANDISING}

\subsection{Aparición del merchandising y confusión terminológica}

Realmente, los comerciantes han practicado merchandising durante milenios. Para abordar la evolución del merchandising desde sus orígenes, habría que remontarse al año 600 a.C., cuando los griegos ya distribuían su excedente de producción a los consumidores mediante pequeñas tiendas minoristas urbanas, que señalizaban con signos y símbolos y en cuyo interior se exhibían los productos colocados en nichos de las paredes.

Esta forma de venta a pequeña escala a través de un mostrador se ha mantenido sin cambios durante siglos, prácticamente sin innovación; en ella los productos se exhibían a través de vitrinas y la argumentación del vendedor es crucial para la venta. En la segunda mitad del siglo XX, el concepto de autoservicio, los grandes almacenes, las nuevas tecnologías de comunicación, las nuevas técnicas de segmentación y el auge de las bases de datos han contribuido a la evolución del comercio al por menor.

En particular, el libre servicio o autoservicio aparece a principios de 1960 en la mayoría de países occidentales, y con él se produce la revolución más trascendental consistente en la presentación del producto al cliente a través de estanterías, cestas y expositores, así como la aplicación de técnicas de marketing en el punto de venta o “merchandising”. Bajo este tipo de 
venta, y para muchos productos de consumo corriente, el papel del vendedor dejó de ser relevante.

A mediados de los 70, términos como packaging, emblistado, o retractilado comienzan a formar parte del argot de la distribución española (Wexter Box, 2005). En esta etapa, podemos hablar del merchandising del fabricante, quien tratará de diseñar productos con envases más atractivos y persuasivos para que puedan venderse por sí solos. Esto hace que se desarrolle la llamada "venta visual” (Zorrilla, 2002), que se extiende también al entorno que rodea a la venta, surgiendo lo que se conoce como merchandising de seducción y que comprende aspectos como el olor, el color, la música o la temperatura.

En el transcurso de los años siguientes, el término "merchandising" cambió bastante en términos de conceptualización y composición. La literatura utiliza varios términos para referirse a las diversas actividades de merchandising (Halpern-Felsher, 2019) asociándolas a las diferentes áreas de marketing, como la distribución (Bar-Zeev et al., 2019), el producto (Braun et al., 2016), la comunicación (Willems et al., 2017) y el precio (Guyader et al., 2017).

En algunos países el término "merchandising” se ha utilizado erróneamente al confundirse con "objetos de merchandising" como camisetas, llaveros o gorras con logotipos de determinadas marcas, series, dibujos animados de éxito comercial. En realidad, en este caso se trataría de "regalos publicitarios", una acción que forma parte de la estrategia comercial de una compañía a través de los derechos de comercialización adquiridos a través de un "contrato de merchandising" (del inglés licensed merchandising) pero que nada tiene que ver con las técnicas de marketing dentro del punto de venta.

Así mismo, en la literatura científica consultada, el término merchandising es usado como sinónimo de comercialización de productos y servicios con un sentido más general. Las expresiones anglosajonas que incluyen el significado de marketing dentro del punto de venta son: 'in-side merchandising', 'self-service merchandising' o 'point-of-sale marketing'. A continuación, se acotará y definirá el término merchandising en un sentido estricto circunscrito al punto de venta.

\subsection{Concepto y tipos de merchandising}

El término merchandising, referido al marketing en el punto de venta, procede del francés merchandise (mercancía) + ing (sufijo del inglés que indica acción o movimiento). Por tanto, lo primero que extraemos del concepto de merchandising es un "movimiento de la mercancía hacia el consumidor”.

El Diccionario Comercial de la L'Académie des Sciences Commerciales lo define como parte del marketing "que abarca los estudios y técnicas implementados por distribuidores y productores (por separado o conjuntamente) que permiten aumentar la rentabilidad del punto de venta y la venta de productos adaptando constantemente la gama de productos a las necesidades del mercado usando una adecuada presentación de los mismos”. Se observa que el merchandising tiende a sustituir la presentación pasiva del producto o servicio, por una presentación activa, apelando a todo lo que puede hacerlo más atractivo: colocación, fraccionamiento, envase y presentación, exhibición, instalación, etc.

La American Marketing Association (AMA), lo considera como un término con significados muy variados y no generalmente adoptado; y recuerda que esta palabra tiene dos significados distintos: a) puede referirse a las actividades promocionales que los fabricantes realizan en las tiendas, tales como expositores específicos para sus artículos, o b) a la gestión y toma de decisiones de los minoristas respecto a un artículo o línea de artículos. 
Con el régimen de libre servicio, aparecen diferentes tipos y evoluciones en merchandising que en ocasiones se dan simultáneamente en el mismo punto de venta. Entre ellos encontramos (Palomares, 2011: 23-27): a) el merchandising visual o de presentación y b) el merchandising estratégico o de gestión. Otros autores reconocieron además la importancia que iba a adoptar en un futuro el "merchandising de seducción” o "sensorial” (Salén, 1987: 106 y ss., citado en Díez and Landa, 1996: 57; Martínez, 2005: 155-171) y el "merchandising de fidelización” (Martínez, 2005: 175). Se trata de estados evolucionados en los procesos comunicativos del punto de venta que pueden aplicarse a cualquier formato y actividad comercial. A continuación, se hace una breve descripción de cada uno de ellos (véase figura 1):

- Merchandising visual. "Visual merchandising” puede ser considerado un concepto moderno, pero en la práctica ha sido utilizado mucho antes de que existiera un término para describirlo. Se centra mayoritariamente en el sentido visual del consumidor para incitar la compra impulsiva (o intuitiva), a través de la disposición interna del establecimiento, la distribución del espacio y la presentación llamativa del surtido. En este tipo de merchandising hay un dominio del fabricante con el uso de acciones de packaging, aunque también técnicas del minorista basadas en la presentación del producto.

- Merchandising estratégico o de gestión. Se apoya en el seguimiento permanente de todas las acciones desarrolladas por la organización, atribuyendo un carácter lúdico a las compras y con la finalidad de conseguir la mayor rentabilidad posible. Además, está centrado en evitar roturas de stocks y en satisfacer permanentemente a un cliente cada vez más exigente.

- Merchandising de seducción o sensorial. Se centra en el eje de animación del merchandising de presentación, con la peculiaridad de que el fabricante y el distribuidor colaboran para llegar al consumidor a través de la seducción. Se definen rasgos que crean atención e interés en una tienda con el objetivo de mejorar la experiencia de compra: ambientes comerciales atractivos, poniendo atención a acciones relacionadas con el color, la música, u otras variables sensoriales o kinestésicas como la iluminación, la temperatura, el olor o el tacto.

- Merchandising de fidelización. Se trata de una nueva etapa evolutiva que consiste en que el punto de venta logre que los consumidores sean capaces de percibirlo como único a través de diferentes acciones y actúen como prescriptores en su entorno más directo.

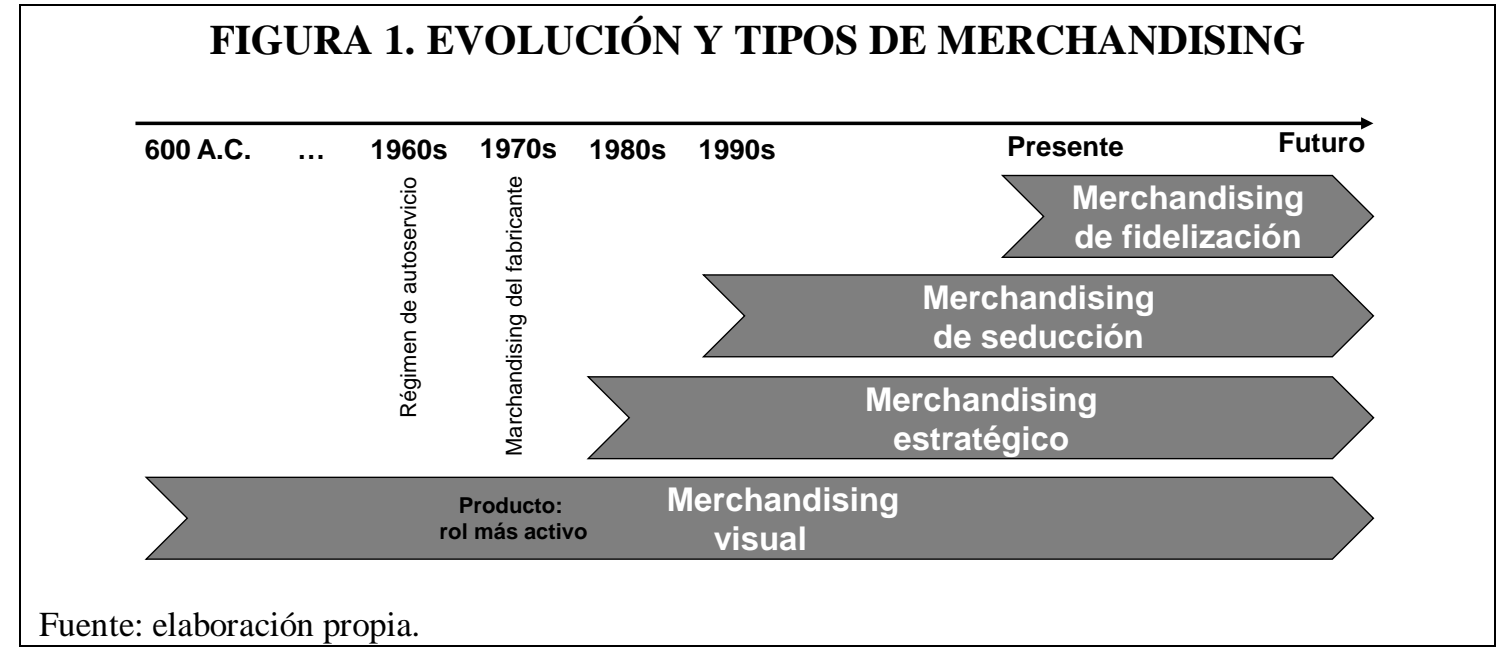


Hasta la fecha, ningún trabajo de los consultados ha realizado un estudio exhaustivo de la investigación académica centrada en esta actividad tan importante para la labor comercial de los minoristas y fabricantes. Una vez descrita la evolución del "self-service merchandising” o "marketing en el punto de venta", nuestro objetivo es detectar aquellas tendencias de investigación que se han confirmado y las que pueden marcar el futuro de las publicaciones sobre esta actividad. Para ello, se ha recurrido a un estudio bibliométrico mediante un análisis co-words a partir de los términos clave extraídos de una búsqueda en la base SCOPUS, incluyendo así revistas de carácter académico y profesional. Esta búsqueda se basó en las expresiones merchandising y marketing en el punto de venta.

\section{METODOLOGÍA}

\subsection{Análisis de co-ocurrencia de palabras clave}

En la presente investigación se ha llevado a cabo un estudio bibliométrico de la investigación sobre merchandising durante el periodo 1960-2019, utilizando el análisis de coocurrencia de palabras clave o co-word analysis. Considerando que los trabajos o documentos publicados en sus respectivas revistas representan "unidades de producción” de conocimiento científico (Talukdar and Arihanan, 2011), el análisis de co-ocurrencia de palabras es una técnica efectiva para mapear las asociaciones entre estas unidades de información (Whittaker, 1989; Callon et al., 1991; Coulter et al., 1998).

Atendiendo al proceso de mapeo de la ciencia o científico que proponen Cobo et al. (2012b), se ha llevado a cabo en primer lugar la obtención de los datos o registros bibliográficos, seguidamente la correspondiente de-duplicación y reducción de los mismos, y la depuración o normalización de palabras clave (este proceso en su conjunto se detalla en el apartado 3.2). Tras esta normalización, se llevan a cabo diferentes análisis de rendimiento que obtienen una aproximación a la evolución dinámica de la investigación sobre merchandising a través del análisis de frecuencias e indicadores para describir las revistas y autores que han publicado un número significativo de publicaciones en este campo de estudio, o el número de publicaciones en las revistas consultadas por año.

La combinación de herramientas de rendimiento y las técnicas de mapas científicos permiten detectar y visualizar los subdominios conceptuales (temas específicos o áreas temáticas generales) así como su evolución (Cobo, 2011; 2012a; 2012b; López-Herrera et al., 2012). Esto es posible mediante la reducción y disposición de las palabras clave en espacios gráficos que reciben el nombre de diagramas estratégicos, así como redes temáticas que ilustran las asociaciones más fuertes entre ellas.

Específicamente, el análisis del contenido de los artículos consultados se basa en una matriz de co-ocurrencia. Se asume que la frecuencia de co-ocurrencia $\left(c_{i j}\right)$ entre dos palabras clave es el número de documentos en los que dos palabras clave aparecen juntas, de entre la colección de documentos analizados. En segundo lugar, se ha de calcular el índice de equivalencia $\left(e_{i j}\right)$ entre palabras clave (Callon et al. 1991), cuya fórmula es:

$$
e_{i j}=\frac{c_{i j}^{2}}{C_{i} \cdot c_{j}},
$$

donde $c_{i j}$ es el número de documentos en los que las palabras clave i y $j$ coocurren y $c_{i}$ y $c_{j}$ representan el número de documentos en los que cada una aparecen. 
Cada red de palabras clave o tema puede ser caracterizada por dos parámetros (Callon et al., 1991; Cobo, 2012b):

- Centralidad: Mide el grado de interacción de un clúster (o conjunto de palabras clave) con otros clústeres y puede ser expresada como:

$$
c=10 \cdot \sum e_{k h},
$$

con $k$ una palabra clave que pertenece al clúster y $h$ una palabra clave perteneciente a otros clústeres.

La centralidad mide la fortaleza de los vínculos externos a otros clústeres o temas (representados por la palabra más central). Este valor puede entenderse como una medida de la importancia de un tema en el desarrollo del campo íntegro de investigación analizado.

- Densidad: Mide la fortaleza interna del clúster y puede ser expresada como:

$$
d=100 \cdot \frac{\sum e_{i j}}{w},
$$

donde i y j son palabras clave que pertenecen al clúster y w el número de palabras en ese clúster.

La densidad mide la fortaleza de los vínculos internos entre todas las palabras clave que forman parte de ese clúster o tema de investigación. Este valor puede ser considerado como una medida del grado de desarrollo del tema bajo estudio.

A partir del rango de estos dos parámetros es posible representar el conjunto de temas sobre un espacio bidimensional denominado diagrama estratégicos (ver ejemplo en figura 2) dando lugar a cuatro grupos (Callon et al. 1991; Cobo, 2012b):

I. Los temas situados en el cuadrante superior derecho están bien desarrollados y son importantes para la estructuración de un campo de investigación. Se trata de temas conocidos como impulsores de la especialidad por su fuerte centralidad y alta densidad.

II. Los temas del cuadrante superior izquierdo presentan vínculos internos bien desarrollados, pero con relaciones externas irrelevantes y de ahí que sean consideradas con una importancia marginal para el área. Estos temas son muy especializados y con un carácter periférico.

III. Los temas del cuadrante inferior izquierdo son marginales y están escasamente desarrollados. Presentan bajos niveles de densidad y centralidad, principalmente representativos de temas emergentes o en desaparición.

IV. Los temas situados en el cuadrante inferior derecho son importantes para el campo de investigación, pero no han sido suficientemente desarrollados pudiéndose catalogar de transversales y básicos o generales. 


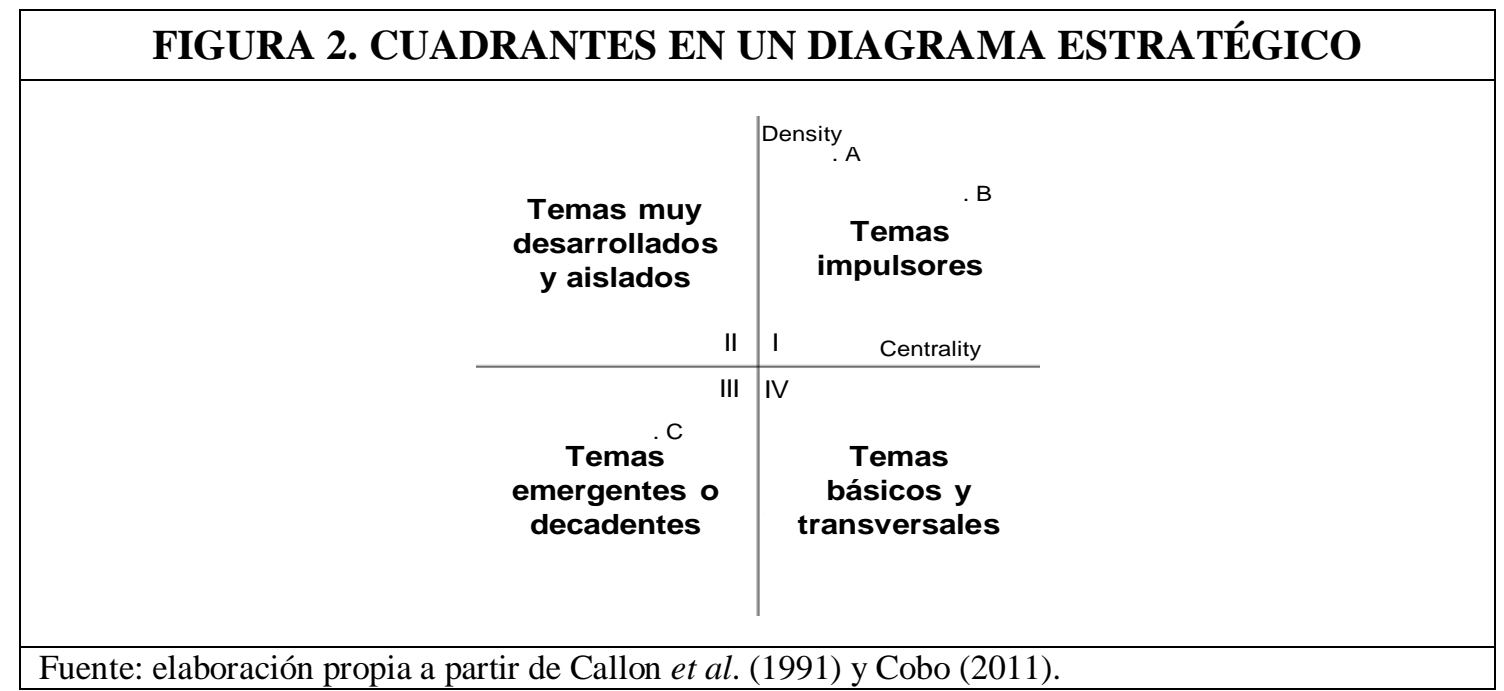

En la fase tercera, las palabras clave y sus interconexiones se representan gráficamente en forma de subredes temáticas (ver ejemplo en figura 3). Estas redes pueden etiquetarse usando el nombre de la palabra clave más significativa para el tema en cuestión y con mayor centralidad (normalmente es la que se sitúa en el centro de la subred). El volumen de las esferas es proporcional al número de documentos correspondientes a cada palabra o el número de veces citados, por ejemplo, y el grosor de las líneas que unen las esferas es proporcional a la frecuencia de co-ocurrencia entre ambas. Un documento es enlazado con otro si contiene al menos dos palabras que están presentes en la red temática (Muñoz-Leiva et al., 2015).

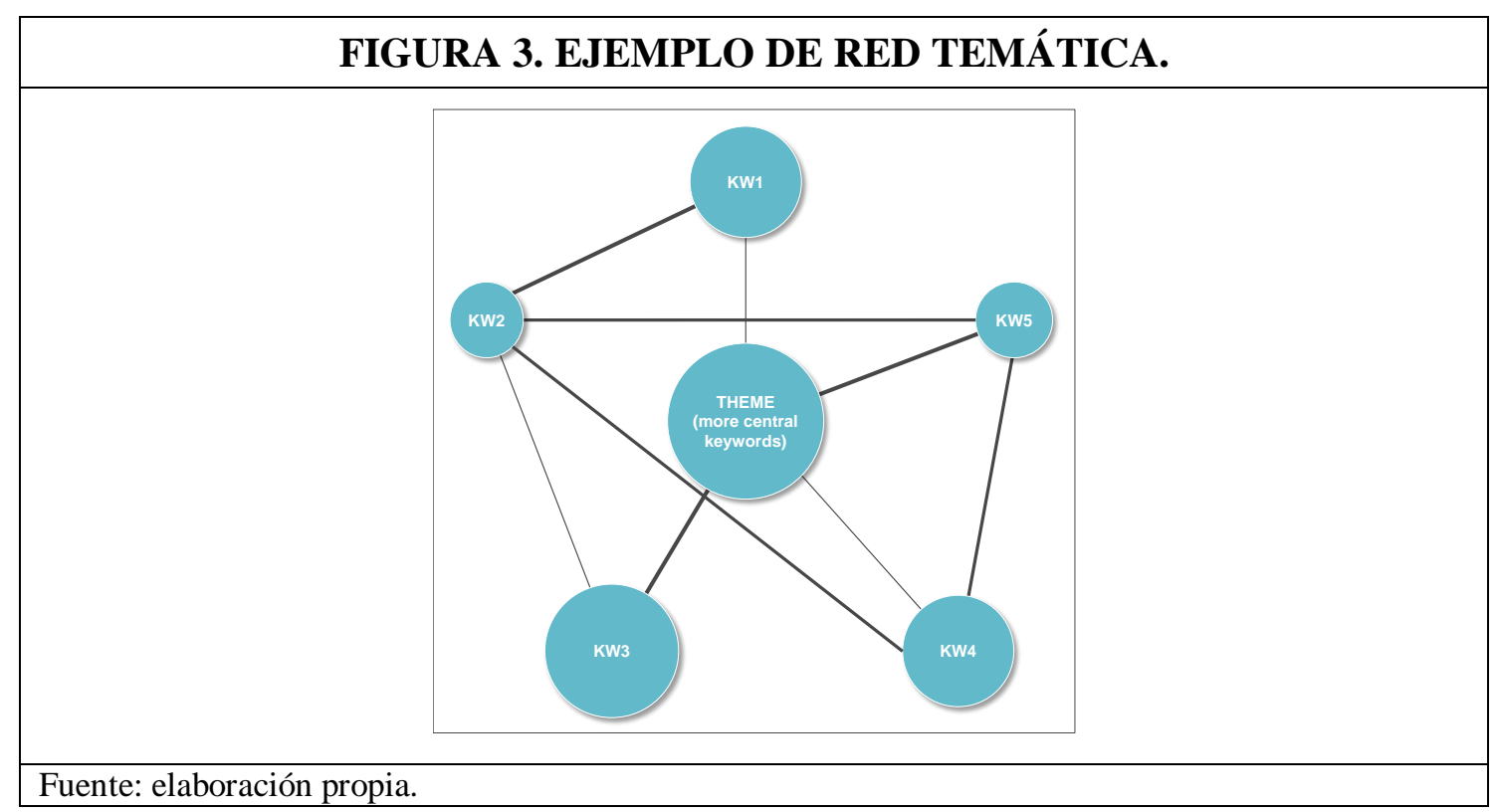
2011).

El análisis co-word ha sido realizado con el software SciMAT ${ }^{4}$ (Cobo et al., 2012b; Sci²s,

${ }^{4}$ SciMAT es un programa de código abierto (GPLv3) desarrollado para realizar un análisis o mapeo científico bajo una perspectiva longitudinal (más información en: http://sci2s.ugr.es/scimat). 


\subsection{Conjunto de datos}

Un total de 1.513 referencias a documentos fueron descargados de la base de datos SCOPUS, incluyendo palabras clave, abstract y medidas de citación; 86 de los cuales se encuentran en open-access.

Scopus es una base bibliográfica propiedad de Elsevier, que contiene resúmenes y citas de artículos de revistas académicas. Scopus ofrece una mayor cobertura geográfica, idiomática que la base de datos Web of Science, así como un número de revistas profesionales, lo que permite realizar comparaciones transnacionales significativas (Delgado y Repiso, 2013). La revisión de Vieira et al. (2009) mostró que Scopus ofrece una mejor cobertura de la literatura de ciencias sociales y en interacción persona-ordenador (IPO; del inglés human-computer interaction -HCI-) y además está bastante equilibrada en términos de áreas temáticas y editores. Estas ventajas formaron la base de nuestra decisión de optar inicialmente por Scopus. La elección de los artículos de revista como unidad de análisis es debido a que la comunidad académica reconoce esas publicaciones como la base de conocimiento más avanzada, actualizada, y ampliamente utilizada al revisar literatura de marketing y más concretamente en el campo de la gestión (Leone et al. 2012).

La consulta en SCOPUS, con una fecha tope de publicación del 31 de diciembre de 2019, fue la siguiente:

\#Query: TS= (“merchandising” OR “in-store marketing” OR "point of sale marketing")

donde el campo TS indica una búsqueda basada en la "temática" ('topic' = 'title' + 'keyword' + 'abstract'). Refinado por tipos de documentos (artículos, encuentros, revisiones u otros).

En este tipo de trabajos, un gran porcentaje de documentos suelen estar referidos a cuestiones procedentes de otras disciplinas por el carácter polisémico de los términos usados o, en ocasiones, por ejemplificar con ellos en el resumen. Por ello, hemos revisado el contenido de los documentos y se han eliminado aquellos que procedían de áreas de ingeniería (ej. cuestiones muy técnicas de equipamiento comercial), trabajos donde el significado de merchandising se refería a la comercialización de un producto o servicio (ej. madera en el Estado de Virginia y Austria), a licencias o derechos de explotación de una marca o personaje animado, actividades de promoción de una serie televisiva, estudios de desempeño de negocios donde el merchandising se analiza de manera muy tangencial o como posible aplicación o estudios aplicados a alumnos de cursos sobre merchandising, entre otros.

Por el contrario, sí se han considerado aquellos trabajos que tratan los siguientes aspectos:

- El “retail marketing” aplicado dentro del punto de venta.

- Visual merchandising.

- Merchandising del fabricante: Acciones de packaging y diseño del producto para aumentar el atractivo del producto en el punto de venta, diseños por ordenador para reducir costes de merchandising, etc.

- Estudios de marcas propias/de distribuidor vs. marcas de fabricante.

- Merchandising exterior: técnicas de escaparatismo, rótulos, logotipos e imagen corporativa del minorista.

- Planificación, medidas de gestión de inventario/surtido (ej. gross margin return on inventory investment -GMROI-), análisis de rotación, y objetivos de ventas. 
- Gestión por categorías, ubicación o posicionamiento estratégico del producto en las estanterías, con o sin uso de planogramas, modelos de gestión del espacio en las estanterías, así como ubicación estratégica del producto en la superficie de venta.

- Componentes, acciones o planificación de PLV o efectos de displays o exhibidores.

- Uso de promociones dentro del establecimiento, uso de cupones de descuento, oferta de productos complementarios o ventas cruzadas en el punto de venta.

- Estudios de neurociencia aplicados a acciones de merchandising.

- Acciones de ambientación o marketing sensorial (música, aromas, iluminación, temperatura, etc.).

- Merchandising virtual, presentación de los productos en sitio web, experiencia en el entorno virtual, etc.

- Realidad virtual (RV) y realidad aumentada aplicada al punto de venta, tecnologías 3 -D, uso robots en la venta minorista, nuevas tecnologías virtuales, y otros avances aplicados al punto de venta.

- Máquinas expendedoras en una superficie comercial.

- Estudios de casos con un singular desarrollo de acciones de merchandising.

- RFID en la venta de productos.

- Estilos de decisión del consumidor con implicaciones en el punto de venta, compras éticas, responsables, impulsivas vs. planificadas, etc.

- Estudios del flujo de circulación de clientes en el establecimiento.

- Etc.

Dado que, en los años 60, con la llegada del libre servicio comienza a hablarse de merchandising como técnica de venta, el periodo de análisis abarcará desde esta década, siendo 691 el total de artículos válidos para el análisis.

La figura 4 recoge el número de artículos consultados para la temática merchandising desde 1960 a 2019.

FIGURA 4. NÚMERO DE ARTÍCULOS POR AÑO EN SCOPUS DESDE 1960 A 2019 SOBRE MERCHANDISING

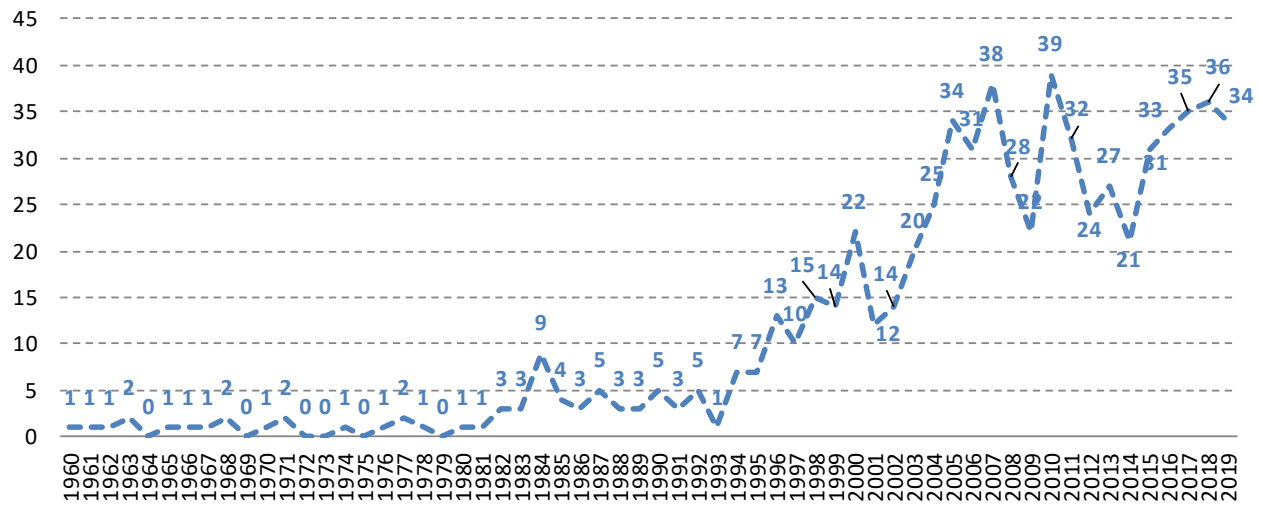

Fuente: elaboración propia. 
Antes de llevar a cabo el análisis co-word, se realiza un proceso de normalización en el que las formas en plural y singular de las palabras son fusionadas (ej. brand en lugar de brands) y los acrónimos son convertidos en sus respectivas palabras clave. También se llevó a cabo una búsqueda semántica, agrupando palabras que estaban escritas de múltiples formas siempre que su significado fuera el mismo. El número total de palabras clave diferentes procesadas fue de 2.418 .

En el análisis co-word, bajo una perspectiva longitudinal, el primer periodo estudiado es usualmente el más amplio para conseguir un número representativo de trabajos publicados. Y el último intervalo de tiempo refleja hacia dónde se orientan los cambios comparándolos con los anteriores periodos y debería permitir una indicación más precisa de las tendencias futuras (emergentes y en declive). En nuestro caso particular se originaron tres conjuntos de datos, uno por cada uno de los periodos 1960-1999, 2000-2009 y 2010-2019, que se corresponden, en el primer periodo, con 40 años, y en los otros dos siguientes, con 10 y 10 años, respectivamente. El número de publicaciones para cada uno de estos periodos es de 133 para el primero (19601999), 246 para el segundo (2000-2009), y 312 (2010-2019) para el tercero.

De esta forma, se pueden construir mapas bibliométricos separados para cada periodo. Los criterios para la generación de la red y reducción de datos han sido: keywords que aparecen en más de 1, 3 y 3 documentos en los periodos 1960-1999, 2000-2009 y 2010-2019, respectivamente. Un valor mínimo de co-ocurrencia de 1,2 y 2, respectivamente. Como medidas bibliométricas de calidad se han utilizado el índice h del keyword, el promedio y la suma de citaciones recibidas hasta la fecha de la descarga de los datos.

\section{RESULTADOS}

\subsection{Análisis de rendimiento}

Las palabras clave asociadas en mayor medida con merchandising son el propio término objeto de consulta, como era de esperar, que aparece referida en 135 artículos (ver tabla 1), seguido de marketing (111), retail (106), sales (68), clothing-industry (49), visual merchandising (37), branding (36), fashion (36), design (33), advertising (30), article(28), human (28), commerce (27), company information (27), electronic commerce (26), packaging (26), economics (24), consumer-behaviour (22), United States (22), Internet (21) o retail-stores (21) con apariciones en más de 20 documentos. 
Muñoz Leiva, F., Rodríguez López, M. E. y Liébana Cabanillas, F.

\begin{tabular}{|c|c|c|c|}
\hline TABLA 1. TÉRMINOS & ASO & CIADOS A MERCHANDISING & \\
\hline TÉRMINO & $\mathbf{n}$ & TÉRMINO & $\mathbf{n}$ \\
\hline MERCHANDISING & 135 & PURCHASING & 17 \\
\hline MARKETING & 111 & CONSUMER-RESEARCH & 16 \\
\hline RETAIL & 106 & $\begin{array}{l}\text { SUPPLY-CHAIN- } \\
\text { MANAGEMENT }\end{array}$ & 16 \\
\hline SALES & 68 & BUSINESS-STRATEGY & 15 \\
\hline CLOTHING-INDUSTRY & 49 & COLOUR & 15 \\
\hline VISUAL-MERCHANDISING & 37 & COMMERCIAL-PHENOMENA & 15 \\
\hline BRANDING & 36 & MANAGEMENT & 15 \\
\hline FASHION & 36 & SOFTWARE & 15 \\
\hline DESIGN & 33 & COMPUTER-AIDED-DESIGN & 14 \\
\hline ADVERTISING & 30 & PRICE & 14 \\
\hline ARTICLE & 28 & PRODUCT-DEVELOPMENT & 14 \\
\hline HUMAN & 28 & TEXTILE-INDUSTRY & 14 \\
\hline COMMERCE & 27 & MARKET & 13 \\
\hline COMPANY-INFORMATION & 27 & VR & 13 \\
\hline ELECTRONIC-COMMERCE & 26 & COST & 12 \\
\hline PACKAGING & 26 & CUSTOMER-SERVICE & 12 \\
\hline ECONOMICS & 24 & DECISION-MAKING & 12 \\
\hline CONSUMER-BEHAVIOUR & 22 & FEMALE & 12 \\
\hline TOBACCO & 22 & COMPETITIVENESS & 11 \\
\hline UNITED-STATES & 22 & INFORMATION-TECHNOLOGY & 11 \\
\hline INTERNET & 21 & INVESTMENT & 11 \\
\hline RETAIL-STORES & 21 & CONSUMER & 10 \\
\hline CUSTOMER-SATISFACTION & 19 & PLANNING & 10 \\
\hline COMPUTER-SIMULATION & 17 & WOMENSWEAR & 10 \\
\hline \multicolumn{2}{|l|}{ TOTAL } & \multicolumn{2}{|l|}{1.853} \\
\hline
\end{tabular}


De los 1.422 autores que han publicado algún trabajo sobre el tema, el más destacado ha sido el periodista y editor J. Ryan con 11 artículos (véase tabla 2), que ha publicado varios trabajos sobre diseño y comercialización visual en tiendas de U.K. y otros países durante los años 2010 y 2011, seguido del profesor G. I. Kunz (6) de la Iowa State University, que ha contribuido significativamente a la teoría y práctica del merchandising, y J. Lee (5), de IBM T.J. Watson Research Center con algunos trabajos sobre clickstream data en tiendas online. En este campo de estudio se observa una gran cantidad de autores con un pequeño número de trabajos (1 ó 2).

\begin{tabular}{|c|c|c|}
\hline \multicolumn{3}{|c|}{$\begin{array}{l}\text { TABLA 2. AUTORES CON MAYOR NÚMERO DE TRABAJOS SOBRE } \\
\text { MERCHANDISING }\end{array}$} \\
\hline Autor & Universidad/Empresa & Frecuencia \\
\hline Ryan, J. & C\&A, United Kingdom (Reino Unido) & 11 \\
\hline Kunz, G.I. & $\begin{array}{l}\text { Dpt. of Textiles and Clothing, Iowa State } \\
\text { University (EEUU) }\end{array}$ & 6 \\
\hline Lee, J. & IBM T.J. Watson Research Center (NY, EEUU) & 5 \\
\hline Buttle, F. & $\begin{array}{l}\text { Dpt. of Hotel, Catering and Tourism } \\
\text { Management, University of Surrey (Reino } \\
\text { Unido) }\end{array}$ & 4 \\
\hline Hoch, S.J. & $\begin{array}{l}\text { Graduate School of Business, University of } \\
\text { Chicago (EEUU) }\end{array}$ & 4 \\
\hline Kim, J. & $\begin{array}{l}\text { Division of Computer Science and Engineering, } \\
\text { Sunmoon University (Korea del Sur) }\end{array}$ & 4 \\
\hline Lee, S.-E. & $\begin{array}{l}\text { Dpt. of Textiles and Clothing, Iowa State } \\
\text { University (EEUU) }\end{array}$ & 4 \\
\hline Rajaram, K. & $\begin{array}{l}\text { Decisions, Operations and Technology } \\
\text { Management, The Anderson School at UCLA, } \\
\text { University of California (EEUU) }\end{array}$ & 4 \\
\hline Speer, J.K. & Northern Group Retail (Canadá) & 4 \\
\hline Walczak, K. & $\begin{array}{l}\text { Poznan University of Economicas and Business } \\
\text { (Polonia) }\end{array}$ & 4 \\
\hline
\end{tabular}


Muñoz Leiva, F., Rodríguez López, M. E. y Liébana Cabanillas, F. Producción científica y evolución conceptual del merchandising durante las últimas seis décadas.

Como se aprecia en la tabla 3, las revistas con un mayor número de trabajos son: International Journal of Retail and Distribution Management (28), Drapers ${ }^{5}$ (27), Apparel (15), Journal of Fashion Marketing and Management (15), Clothing and Textiles Research Journal (13), TextilWirtschaft (10) y Tobacco Control (10).

\begin{tabular}{|c|c|}
\hline \multicolumn{2}{|c|}{ TABLA 3. REVISTAS MÁS PRODUCTIVAS EN MERCHANDISING } \\
\hline Revistas & Frecuencia \\
\hline International Journal of Retail and Distribution Management & 28 \\
\hline Drapers & 27 \\
\hline Apparel & 15 \\
\hline Journal of Fashion Marketing and Management & 15 \\
\hline Clothing and Textiles Research Journal & 13 \\
\hline Journal of Retailing & 10 \\
\hline TextilWirtschaft & 10 \\
\hline Tobacco Control & 10 \\
\hline Journal of Global Fashion Marketing & 9 \\
\hline Journal of Retailing and Consumer Services & 9 \\
\hline American Journal of Agricultural Economics & 8 \\
\hline Fashion Business International & 8 \\
\hline LDB Interior Textiles & 8 \\
\hline AATCC Review & 7 \\
\hline European Journal of Marketing & 7 \\
\hline Home Textiles Today & 7 \\
\hline Marketing Science & 7 \\
\hline Official Board Markets & 7 \\
\hline International Paper Board Industry & 6 \\
\hline Cornell Hotel and Restaurant Administration Quarterly & 5 \\
\hline Journal of Business Research & 5 \\
\hline
\end{tabular}

\footnotetext{
${ }^{5}$ Drapers fue fundada en 1887 para cubrir el comercio minorista de prendas de vestir, especialmente orientado a mujeres; pero en la actualidad abarca todo el sector minorista, incluidos temas minoristas más amplios como la transformación digital, los avances tecnológicos y la gestión de las cadenas de suministro.
} 


\subsection{Análisis de contenido del periodo 1960-1999}

En el primer periodo (1690-1999) un total de 133 trabajos fueron publicados por la comunidad interesada en el marketing en el punto de venta. En los años sesenta ya comienzan a aparecer estudios de comercialización de productos lácteos a través de establecimientos minoristas y el uso de marcas en este sector. Otros trabajos se centraron en la evaluación de las preferencias de marcas, su modelización, así como los efectos producidos por los exhibidores o expositores (displays) en los supermercados. Algunos de los primeros experimentos se centraban en controlar el precio, la calidad del espacio de visualización y la localización del display para aislar el efecto de la preferencia de marca sobre las compras del consumidor (ej. Padberg et al., 1967). Algunos trabajos revisaron técnicas de venta personal, iluminación, diseño de sistemas de códigos de barras, envases de productos alimenticios o diseño asistido por ordenador de productos o envases.

En los ochenta se aprecian diferentes estudios de corte y presentación de carnes, nuevamente merchandising en la industria láctea, así como descripciones de los primeros macrocentros comerciales (como el C.C. Northgate, a las afueras de Seattle, EEUU) de ámbito regional. Algunos trabajos también analizaron el efecto de la fijación de precios o el efecto visual de los empaquetados con etiqueta adhesiva aplicada a blisters. Otros propusieron medidas de retorno de la inversión del inventario. En esta década comienza a desarrollarse una teoría del merchandising basándose en la psicología ambiental, que estudia los efectos de los entornos físicos en el comportamiento humano. La teoría propone que el comportamiento de la tienda, evaluado en términos de evasión/acercamiento, es determinado por respuestas emocionales a los estímulos de merchandising, atenuados o potenciados por los conjuntos de respuestas propias del consumidor (Buttle, 1984).

En la década de los noventa aparece un mayor número de trabajos publicados, principalmente en la segunda mitad, y con ello la diversificación de temáticas bajo estudio. Continúan los estudios de corte de carne para su venta, pero encontramos nuevos estudios sobre: centros comerciales, tipos de stocks, planificación y gestión del surtido (posición de las categorías en la superficie y familias en el lineal), forma de presentación de los productos más eficaz, diversificación de la oferta de productos no alimenticios, promociones en puntos de venta, acciones publicitarias en los estancos y máquinas expendedoras, y la regulación de la venta de tabaco, así como atmósfera del establecimiento y su efecto sobre la generación de emociones y experiencia de compra.

Para analizar este periodo desde una forma conceptual y agregada se muestran dos diagramas estratégicos en la figura 5. Los temas impulsores del periodo 1960-1999 y por tanto de mayor centralidad y densidad son toma de decisiones ${ }^{6}$ y a la organización del punto de venta. Los términos como moda y humano también aparecieron en algunos trabajos (3 y 2 trabajos respectivamente). Gestión y organización (es decir, merchandising de gestión) y marketing aparecen en una posición que indica una gran fortaleza de los vínculos externos a otros temas, así como un elevado número de apariciones y citaciones. Se trata de temas que son importantes para la estructuración del merchandising como campo de investigación.

Diseño y productos del consumidor muestran cierta fortaleza de los vínculos internos o desarrollo entre todas las palabras clave que describen el tema de investigación (densidad), con un mayor número de citas en el primer caso.

Información de la compañía bajo estudio y acciones de packaging por el fabricante son temas emergentes, como se comprobó en los siguientes periodos. En el primer caso,

\footnotetext{
${ }^{6}$ Los temas o temáticas aparecen en mayúscula.
} 
información de la compañía, apareció en un 62,9 por cien de los trabajos del segundo periodo de tiempo analizado (2000-2009) y en un 22,2 por cien de los del tercer periodo (2010-2019). Packaging, por su parte, se encuentra en un 61,3 por cien de los trabajos del segundo periodo y en un 12,9 por cien de los del tercero.

Un examen pormenorizado de los trabajos extraídos desvela que toma de decisiones es un tema que se ha desarrollado a finales del periodo a través de un grupo de investigación centrado en realizar análisis comparativos del desempeño o competitividad de diferentes empresas de servicios (diseño, publicidad, consultoras, investigación de mercados, etc.) de las regiones de Gales, Escocia, Irlanda o Inglaterra. Con respecto a la temática de organización, tal y como refleja su alto grado de centralidad, ha estado vinculada a una gran variedad de temáticas tales como relaciones ${ }^{7}$, incertidumbre u organización del canal, pero bajo una perspectiva de la teoría de los costes de transacción.

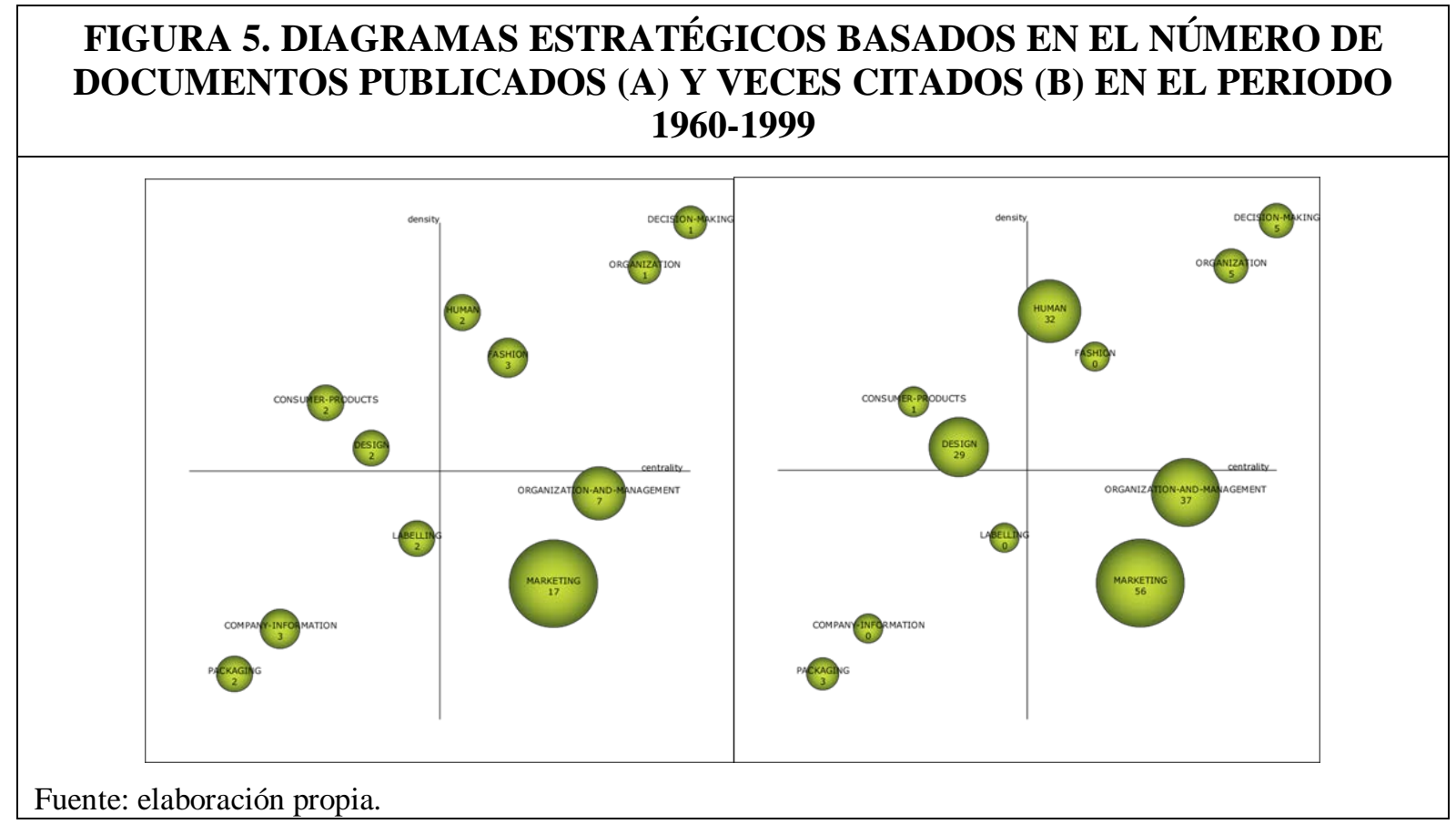

\subsection{Análisis de contenido del periodo 2000-2009}

Con el nuevo siglo, aumenta el número de publicaciones en materia de merchandising, así como la diversidad de tópicos abordados. En total, se publicaron 246 trabajos indexados en la base Scopus. Entre otras temáticas, encontramos el merchandising en el sector de la moda, cocreación de diseños en colaboración con los clientes, creación de artículos personalizados, marca privada o "de distribuidor" en el sector del retail, el uso de productos descontados o "gancho", los programas de exhibición (con carteles, displays, pantallas, etc.), estudio de los flujos de clientes en tiendas o casos de empresas singulares en sus acciones de merchandising (Mark \& Spencer, Matalan, H\&M, Zara, etc.). Las nuevas tecnologías se introducen en el sector y aparecen varios trabajos sobre el diseño asistido por ordenador (CAD- Computer Aided Design), sobre merchandising electrónico (e-merchandising) y la forma de disponer los

\footnotetext{
${ }^{7}$ En cursiva se marcan las palabras que aparecen en alguna red temática del análisis co-word.
} 
productos en el sitio web (ej. Romero et al., 2009) o de generar fidelidad, estrategia de marca de los fabricantes en sus negocios on line, aplicaciones CRM (Customer Relationship Management) o sistemas/etiquetas RFID (Radio Frequency Identification), entre otros estudios.

Para este segundo intervalo (2000-2009) se han extraído los siguientes diagramas estratégicos (Figura 6). Un examen conceptual de los mismos muestra que los artículos o productos, la ropa de hombre y el propio sector al por menor o retail han centro gran atención de los estudios en materia de merchandising y constituyen temas impulsores de esta disciplina (con fuerte centralidad y alta densidad); como podría resultar lógico a tenor de la consulta realizada. En el primer caso se trata de un término genérico aplicable al estudio de productos gancho y sobre todo el consumo de cigarrillos (5 de los 7 trabajos referidos a artículos). Se trata de una palabra clave con muchas citas (319), superado solo por retail (391).

Diferentes aspectos económicos, e incluso la satisfacción del consumidor, son temas importantes para la investigación en marketing, pero no han sido suficientemente desarrollados en este campo al presentar una menor fortaleza de los vínculos internos con las otras palabras clave que co-ocurren con ellos. En realidad, se trataría de temas básicos o generales y transversales para el merchandising durante esta década.

Las simulaciones por ordenador en este campo (con CAD, tecnologías 3-D, realidad virtual, etc.) han sido bien desarrollados (internamente, dada su alta densidad) aunque presentan relaciones externas irrelevantes (en términos relativos). La industria del papel y celulosa ha estado presente en estudios sobre empresas de troquelado, tendencias en el sector, o soluciones (tipo RFID) aplicables a este subsector. Estos temas son muy especializados y con un carácter periférico y por tanto con una importancia marginal para el área.

Para este periodo, la industria textil o de la ropa de vestir y el comercio electrónico resultan aún marginales y además escasamente desarrollados (baja densidad y centralidad) en este campo de conocimiento. Como se comprobarán en el siguiente periodo, representan temas decadentes que mantienen aproximadamente la misma posición.

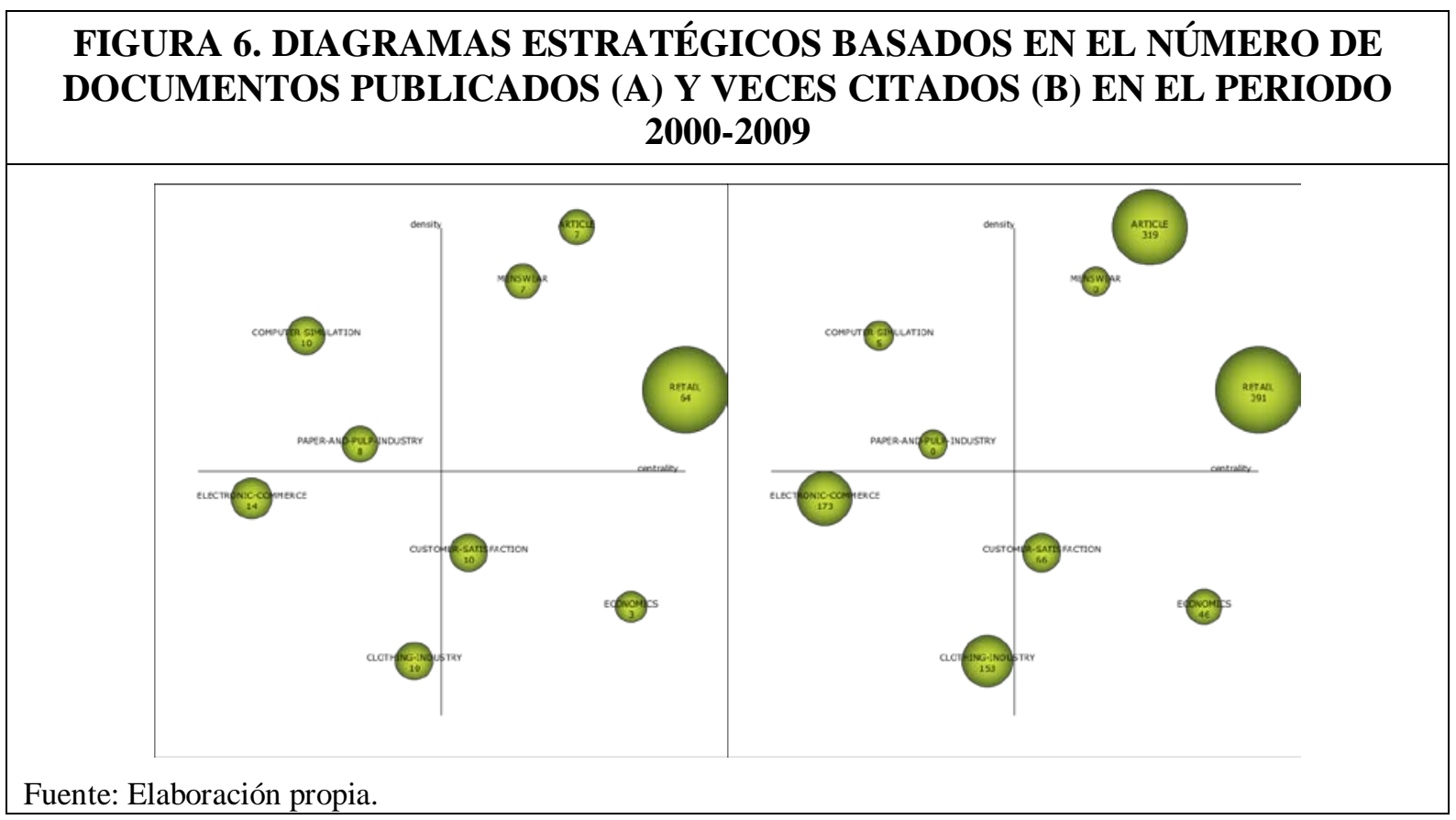




\subsection{Análisis de contenido del periodo 2010-2019}

En la década pasada, se han publicado un total de 312 trabajos y en línea con la década anterior, han aparecido un gran número de artículos en materia de merchandising visual en general, escaparatismo, diseño del envase (packaging) y diseño del propio producto (ej. textiles y ropa), técnicas de presentación de productos, venta cruzada, realidad virtual y aumentada aplicada al punto de venta, co-merchandising (fabricante-minorista), uso de herramientas para la generación de planogramas, experiencias para mejorar la experiencia de compra, merchandising virtual, RFID, y efecto de variables ambientales en el comportamiento de compra.

En primer lugar, observamos como el marketing orientado al sector masculino, los factores demográficos, nuevamente los artículos, el comportamiento del consumidor y el fenómeno comercial han constituido recientemente temas impulsores muy bien desarrollados en estudio del merchandising (véase figura 7). Un análisis detallado de los trabajos consultados pone de manifiesto que varias investigaciones sobre aquellos factores o variables de clasificación del sujeto han sido aplicadas al consumo de alimentos orgánicos, el marketing del tabaco en los puntos de venta o el consumo de carne en el sector minorista. El tema artículos sigue manteniendo la posición privilegiada de los últimos años, pero en esta década deja de centrarse casi exclusivamente en el sector de venta de tabaco y lo hace también en estrategias de merchandising innovadoras y descuentos en bebidas y alimentos (ej. ternera). Otras áreas como el consumo de alimentos orgánicos y la estrategia de educación del consumidor en el proceso de compra de alimentos y bebida han sido abordados bajo los fundamentos de la sicología.

La información sobre una compañía, y principalmente la gestión, aparecen ahora con un carácter periférico y muy especializado, aunque con una importancia marginal para el área (baja centralidad). Estas palabras clave parecen indicar que en los próximos años se desarrollarán trabajos con carácter más cuantitativo y de carácter estratégico.

En este periodo la publicidad, incluso el retail, aparece como temas generales y transversales a otras temáticas, en particular, retail, pierde fortaleza de sus vínculos externos (centralidad) y desarrollo interno (densidad).

Los análisis de costes han ido en aumento en este último periodo, si comparamos con los anteriores, por tanto, podría considerado un tema emergente. El estudio de la industria textil, ha perdido importancia en términos cuantitativos (número de documentos) y calidad (citación), si lo comparamos con el periodo anterior. Los temas comercio electrónico, displays y/o merchandising visual resultan aún marginales, y relativamente poco citados. Por tanto, previsiblemente se convertirán con el paso del tiempo en temas en desaparición, o emergentes en algún caso. En el caso del merchandising visual, se trata de un tipo de merchandising cuyo primer trabajo data de 1998 y, el siguiente, de 2002. El primer trabajo de Lawrie (1998) se centra en analizar el papel de la publicidad (en particular, carteles y expositores publicitarios) en puntos de venta textil. Otros trabajos posteriores, analizan a) la agrupación de productos similares y su superposición, b) linealidad de los displays, c) uso de accesorios, (d) densidad de productos, así como e) su aplicación al contexto del comercio electrónico. Un trabajo reciente (Widyastuti, 2018) analiza el papel del merchandising virtual, la atmósfera del establecimiento y la marca del distribuidor en la compra impulsiva. En particular, el 11,8 por cien de los trabajos sobre merchandising visual aparecen publicados en el segundo periodo y el 85,3 por cien en este último periodo; por tanto, lo consideramos como un tema emergente. 


\section{FIGURA 7. DIAGRAMAS ESTRATÉGICOS BASADOS EN EL NÚMERO DE DOCUMENTOS PUBLICADOS (A) Y VECES CITADOS (B) EN EL PERIODO 2010-2019}

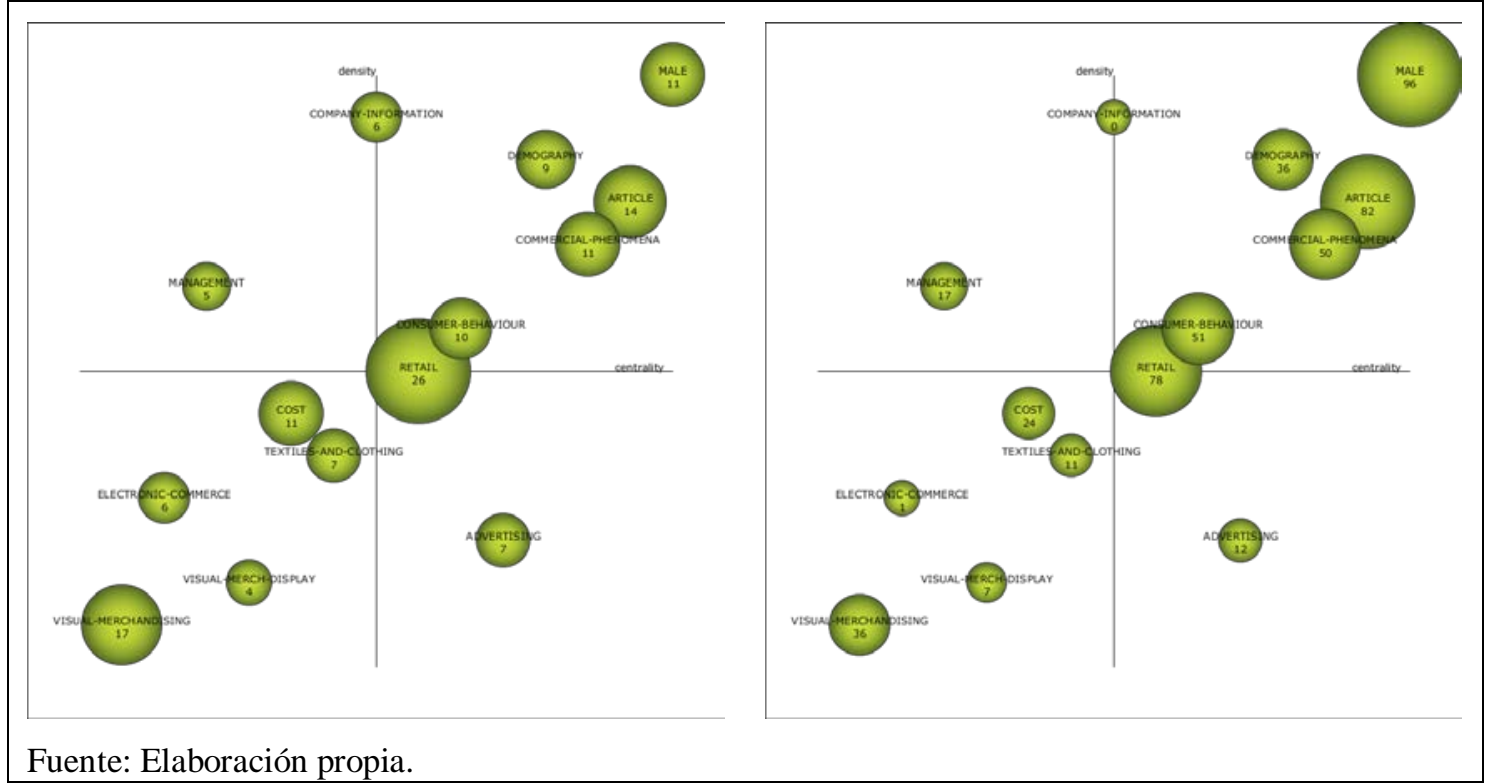

\subsection{Evolución conceptual del merchandising}

Para analizar la evolución conceptual de temas más recurrentes abordados por la comunidad de estudiosos sobre este tema, se presenta a continuación el mapa longitudinal basado en la cantidad de publicaciones del periodo estudiado. En este mapa el volumen de las esferas es proporcional al número de documentos publicados y asociados a cada tema.

Si bien el primer trabajo sobre merchandising visual data de 1998 (Lawrie, 1998), tal y como se aprecia en la siguiente ilustración (figura 8), el merchandising visual ha sido objeto de publicación principalmente en el nuevo milenio; concretamente a partir de 2002 y con una fuerte aplicación a la industria textil. Otros términos recurrentes usados en artículos publicados en los dos últimos periodos han sido artículos (article) o industria de la ropa (clothing industry y textiles and clothing), incluyendo en el último periodo el uso de displays en el merchandising visual. Retail es un término que apareció en el segundo periodo en un gran número de documentos que fueron altamente citados y ha estado asociado más recientemente a la disciplina del marketing en general y el merchandising en particular. Una temática que también tuvo cierta presencia durante las dos últimas décadas fue la aplicación de los fundamentos del merchandising al caso del comercio electrónico.

El estudio del fenómeno comercial en el último periodo ha mantenido un enfoque economista heredado del periodo intermedio. A lo largo del tiempo, los productos, su coste y la satisfacción generada en el consumidor han mantenido cierta vinculación.

A pesar de la diversidad de temas recurrentes de cada periodo, se puede concluir que no existe ni un solo tema fuertemente cohesionado, es decir, que haya permanecido en las tres etapas. Esto es una muestra de la fuerte fragmentación, así como la escasa centralidad y, por tanto, escaso desarrollo de las diferentes temáticas en el desarrollo del campo íntegro de investigación analizado. 


\section{FIGURA 8. MAPA DE EVOLUCIÓN* SEGÚN NÚMERO DE DOCUMENTOS CADA TEMA}

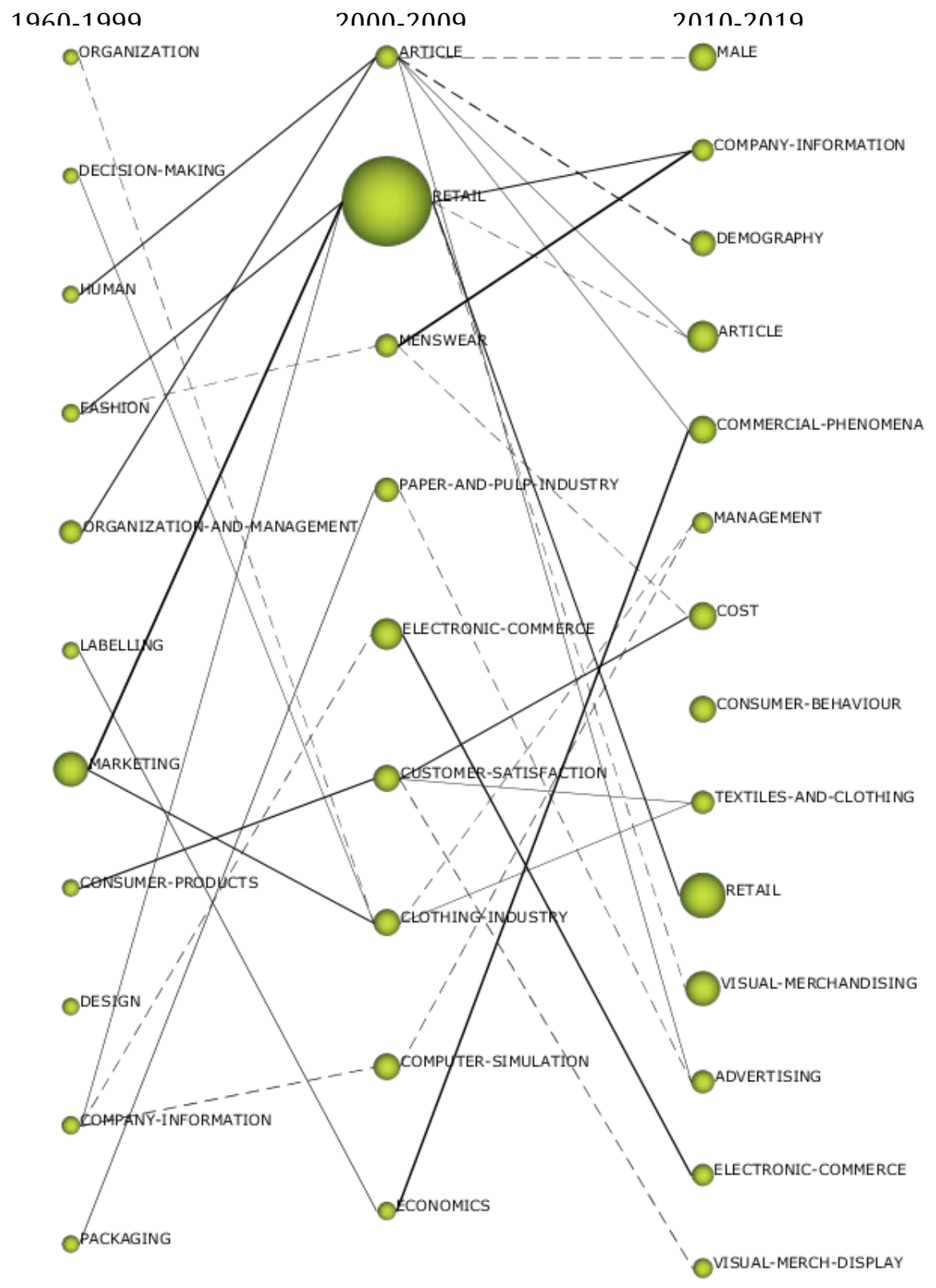

Fuente: Elaboración propia.

* Línea continua: ambos clústeres (o temas) comparten la misma palabra clave más central (nombre usado para el tema), o esta palabra está incluida en el otro clúster.

Línea discontinua: los clústeres comparete alguna palabra clave.

La anchura de la línea es proporcional al índice de Jaccard. 


\section{CONCLUSIONES}

En el presente artículo aparecen los principales resultados de un análisis bibliométrico aplicado a la comunidad académica y profesional interesada por el estudio del merchandising. Tras la correspondiente revisión y eliminación de los trabajos que abordaban otras cuestiones diferentes, algunas keywords tuvieron que recodificarse o se normalizaron bajo un criterio homogéneo de clasificación. Finalmente, casi 700 artículos han sido procesados. El análisis coword aplicado permite visualizar la estructura conceptual de esta área de conocimiento a partir de las keywords de los artículos revisados, siendo los resultados de gran utilidad para la investigación empírica sobre marketing en general, o específicamente, sobre merchandising o marketing en el punto de venta.

En primer lugar, encontramos que a lo largo del periodo estudiado (1960-2019), el número de temas ha crecido a causa del incremento en el número de artículos publicados (y creación de nuevas revistas) en este campo de conocimiento. Pero este hecho no ha seguido una progresión lineal, sino exponencial sobre todo a durante la segunda mitad de los años noventa y comienzos del primer decenio. Este hecho ha provocado que, en los dos últimos periodos (2000-2009 y 2010-2019), el número de artículos hayan sido agrupados en temas de mayor tamaño.

Los resultados de este análisis bibliométrico muestran que la industria textil fue un sector fuertemente estudiado en la década de los noventa, si bien, en los últimos años, ha ido perdiendo importancia en términos cuantitativos y de calidad. La presentación de datos e informaciones sobre las compañías bajo estudio presenta un marcado carácter periférico y especializado, aunque con una importancia marginal para el área. Con el paso del tiempo, aportar información de las empresas ha ido mejorando la fortaleza de los vínculos internos de las palabras clave con las que se vincula, es decir, su grado de desarrollo.

El estudio de los simuladores por ordenador, se trata de un tema periférico durante en el segundo periodo con diferentes programas como CAD e incluso la gestión de la cadena de suministro aplicados al sector textil. Un análisis más pormenorizado de los trabajos publicados muestra que el futuro pasará por la aplicación de la realidad virtual (VR) y realidad aumentada al caso del e-merchandising.

El estudio del fenómeno comercial, las variables personales socio-económicas, la aplicación de la psicología y la presentación de estadísticas sobre consumo y casos de empresas constituyen temas impulsores muy bien desarrollados en el estudio del merchandising. Recientemente la investigación sobre variables de clasificación del sujeto ha sido aplicada al consumo de alimentos orgánicos o el marketing del tabaco en los puntos de venta. Aproximadamente la mitad de los documentos con estadísticas y datos numéricos están referidos también a acciones de merchandising en la venta de tabaco y las grandes transformaciones que ha sufrido este sector en los últimos años. Estos resultados, parecen indicar que en los próximos años se desarrollarán trabajos en este ámbito con un enfoque más cuantitativo. Otra gran cantidad de artículos publicados se han centrado en demografía de los consumidores (género y otras variables demográficas) así como el propio fenómeno comercial. Estos temas constituyen cuestiones impulsoras en la década que dejamos atrás, contribuyendo con ello a la estructuración de la investigación académica y profesional del merchandising en el presente decenio.

Por último, el merchandising visual, por su parte, es una temática emergente en la literatura académica que irá integrando el papel del merchandising virtual, la atmósfera del establecimiento, la marca del distribuidor en la compra impulsiva y técnicas de escaparatismo. 


\section{LIMITACIONES Y FUTURAS LÍNEAS DE INVESTIGACIÓN}

El presente estudio ha alcanzado el objetivo de identificar la estructura de la red de temas de investigación sobre merchandising o marketing en el punto de venta. Sin embargo, los autores han encontrado varias dificultades debido a los sesgos que se derivan de los análisis de este tipo.

En primer lugar, una de las limitaciones es que la resolución del método aplicado depende de los criterios establecidos en el proceso de homogeneización de datos, así como los umbrales y medidas para la reducción y normalización de la red o para la extracción de los diagramas estratégicos y redes temáticas. Aunque, los autores ampliaron estos umbrales anteriores sin identificar cambios significativos en las estructuras conceptuales identificadas, las soluciones finales dependen parcialmente de las decisiones técnicas tomadas. En cualquier caso, el análisis realizado permite la discusión sobre tendencias generales ampliamente aceptadas por la comunidad científica para ser legitimados ya que el procedimiento excluye los temas de importancia marginal, y aclara la interpretación de los resultados.

Los autores sugieren extender la investigación futura mediante otros procedimientos, y el uso de otras bases bibliográficas como puede ser la de Web of Science para un análisis más exhaustivo. Además, podría ser interesante tener en cuenta otros tipos de documentos como aquellos generados en centros de investigación de prestigio, manuscritos pre-publicados disponibles solo online o ponencias de congresos.

Además, los hallazgos sugieren que la investigación existente no es exhaustiva, lo que indica que se necesita un mayor esfuerzo investigador en cada uno de los subdominios o corrientes de investigación reconocidas para obtener una mejor comprensión de los aspectos cruciales del merchandising. Estos resultados responden al reclamo de los autores a una mayor investigación dirigida a aclarar los fundamentos teóricos de este campo de estudio, que aún puede considerarse en desarrollo.

Las futuras líneas de trabajo se han explicado en función de las limitaciones reconocidas en los numerosos artículos estudiados, aunque estas líneas podrían matizarse, por ejemplo, mediante la creación de un panel de expertos en el tema. No obstante, las ideas presentadas en este documento, así como la estructura conceptual bajo una perspectiva longitudinal contribuyen a avanzar en el conocimiento y la comprensión de la investigación académica sobre merchandising.

\section{BIBLIOGRAFÍA}

Bailón-Moreno, R., Jurado-Alameda E. y Ruiz-Banos, R. (2006): “The scientific network of surfactants: Structural analysis”, Journal of the American Society for Information Science and Technology, vol. 57, $\mathrm{n}^{\circ}$ 7, pp. 949-60.

Bar-Zeev, Y., Levine, H., Rubinstein, G., Khateb, I. y Berg, C.J. (2019): “IQOS point-of-sale marketing strategies in Israel: A pilot study”, Israel Journal of Health Policy Research, vol. 8, $\mathrm{n}^{\mathrm{o}} 11$, pp. 1-4.

Börner, K., Huang, W., Linnemeier, M., Duhon, R. J., Phillips, P., Ma, N., Zoss, A.M., Guo, H. y Price, M.A. (2010):”Rete-netzwerk-red: analyzing and visualizing scholarly networks using the Network Workbench Tool”, Scientometrics, vol. 83, n 3, pp. 863876.

Braun, S., Kollath-Cattano, C., Barrientos, I., Mejia, R., Morello, P., Sargent, J.D. y Thrasher, J.F. (2016): "Assessing tobacco marketing receptivity among youth: integrating point of sale marketing, cigarette package branding and branded merchandise", Tobbaco Control, vol. 25, $\mathrm{n}^{\circ}$ 6, pp. 648-655. 
Buttle, F. (1984): “How merchandising works?”, International Journal of Advertising, vol. 3, $\mathrm{n}^{\circ}$ 2, pp. 139-148.

Callon, M., Courtial, J. P. y Laville, F. (1991): "Co-word analysis as a tool for describing the network of interactions between basic and technological research - the case of polymer chemistry", Scientometrics, vol. 22, no 1, pp. 155-205.

Cobo, M. J., López-Herrera, A. G., Herrera, F. y Herrera-Viedma, E. (2012a): “A note on the its topic evolution in the period 2000-2009 at T-ITS”. IEEE Transactions on Intelligent Transportation Systems, vol. 13, n⿳⿲丶丶㇒一 1 , pp. 413-420.

Cobo, M. J., López-Herrera, A. G., Herrera-Viedma, E. y Herrera, F. (2011): “An approach for detecting, quantifying, and visualizing the evolution of a research field: A practical application to the fuzzy sets theory field”, Journal of Informetrics, vol. 5, n 1, pp. 146166.

Cobo, M. J., López-Herrera, A. G., Herrera-Viedma, E., y Herrera, F. (2012b): “SciMAT: A new science mapping analysis software tool”, Journal of the American Society for Information Science and Technology, vol. 63, $\mathrm{n}^{\circ}$ 8, pp. 1609-1630. https://doi.org/10.1002/asi.22688.

Cornelius, B. y Persson, O. (2006): “Who’s who in venture capital research”, Technovation, vol. 26, $\mathrm{n}^{\mathrm{o}} 2$, pp. 142-150.

Coulter, N., Monarch, I. y Konda, S. (1998): "Software engineering as seen through its research literature: A study in co-word analysis", Journal of the American Society for Information Science, vol. 49, $\mathrm{n}^{\circ} 13$, pp. 1206-1223.

Culnan, M. (1986): “The intellectual development of management information systems”, Management Science, vol. 32, $\mathrm{n}^{\circ}$ 2, pp. 156-172.

Díez de Castro, E. y Landa Bercebal, F. J. (1996): Merchandising. Teoría y Práctica, Ed. Pirámide, Madrid.

Echchakoui, S. y Mathieu, A. (2008): "Marketing trends: Content analysis of the major journals (2001-2006)", Proceedings of Administrative Sciences Association of Canada, Nova Scotia, Mai 27-28, pp. 114-126.

Guyader, H., Ottosson, M. y Witell, L. (2017): "You can’t buy what you can't see: Retailer practices to increase the green Premium”, Journal of Retailing and Consumer Services, 34, pp. 319-325.

Halpern-Felsher, B. (2019): "Point-of-sale marketing of heated tobacco products in Israel: cause for concern”, Israel Journal of Health Policy Research, vol. 8, $\mathrm{n}^{\circ}$ 1, pp.47.

He, Y. y Hui, S.C. (2002): "Mining a web citation database for author cocitation analysis", Information Processing and Management, $\mathrm{n}^{\circ}$ 38, pp. 491-508.

L'Académie des Sciences Comerciales (2018): "Definition of merchandising" in Le Dictionnaire Commercial, disponible on line en: http://www.academie-des-sciencescommerciales.org/.

Leone, R.P., Robinson, L.M., Bragge, J. y Somervuori, O. (2012): “A citation and profiling analysis of pricing research from 1980 to 2010”, Journal of Business Research, $n^{\circ}$ 65, pp. 1010-1024.

Leydesdorff, L. y Zhou, P. (2008): “Co-word analysis using the Chinese character set”, Journal of the American Society for Information Science and Technology, vol. 59, n $\mathrm{n}^{\circ}$ 9, pp. 15281530.

López-Herrera, A. G., Cobo, M. J., Herrera-Viedma, E. y Herrera, F. (2010): “A bibliometric study about the research based on hybridating the fuzzy logic field and the other computational intelligent techniques: A visual approach”, Internacional Journal of Hybrid Intelligent Systems, vol. 17, nº 7, pp. 17-32. 
López-Herrera, A. G., Cobo, M. J., Herrera-Viedma, E., Herrera, F., Bailón, R. y JiménezContreras, E. (2009): "Visualization and evolution of the scientific structure of fuzzy sets research in Spain”, Information Research, vol. 14, n 4, paper 421.

Martínez Martínez, I. J. (2005): La comunicación en el punto de venta, ESIC, Madrid.

McAlpine, I. A. (1960): "Marketing of dairy products in Scotland”, International Journal of Dairy Technology, vol. 13, no 3, pp. 168-176.

Morris, S.A. y Van Der Veer Martens, B. (2008): “Mapping research specialties”, Annual Review of Information Science and Technology, vol. 42, n 1, pp. 213-95.

Muñoz-Leiva, F., Porcu, L. y del Barrio-García, S. (2015): "Discovering prominent themes of Integrated Marketing Communication research from 1991 to 2012: A co-word analytic approach”, International Journal of Advertising, vol. 34, nº 4, pp. 678-701.

Muñoz-Leiva, F., Sánchez-Fernández, J., Liébana-Cabanillas, F.J. y López-Herrera, A.G. (2012a): "Applying an automatic approach for showing up the hidden themes in financial marketing research (1961-2010)”, Expert Systems with Applications, vol. 39, no 12, pp. 11055-11065.

Muñoz-Leiva, F., Sánchez-Fernández, J., Liébana-Cabanillas, F.J. y Martínez-Fiestas, HM. (2013): "Detecting salient themes in financial marketing research from 1961 to 2010", The Service Industries Journal, vol. 33, n 9-10, pp. 925-940.

Muñoz-Leiva, F., Viedma-del Jesús, M.I., Sánchez-Fernández, J. y López-Herrera, A.G. (2012b): “An application of co-word analysis and bibliometric maps for detecting the most highlighting themes in the consumer behaviour research from a longitudinal perspective”, Quality \& Quantity, vol. 46, nº 4, pp. 1077-1095.

Padberg, D. I., Walker, F. E. y Kepner, K. W. (1967): “Measuring consumer brand preference”, American Journal of Agricultural Economics, vol. 49, n 3, pp. 723-733.

Palomares, R. (2011): Merchandising Merchandising: Teoría, práctica y estrategia, $2^{\mathrm{a}}$ edición, ESIC, Madrid.

Ramos-Rodríguez, A.R. y Ruíz-Navarro, J. (2004): "Changes in the intellectual structure of strategic management research: A bibliometric study of the Strategic Management Journal, 1980-2000”, Strategic Management Journal, n² 25, pp. 981-1004.

Rodríguez-López, M.E., Alcántara-Pilar, J.M., Del Barrio-García, S. y Muñoz-Leiva, F. (2019): "A review of restaurant research in the last two decades: A bibliometric analysis", International Journal of Hospitality Management, In press, DOI: 10.1016/j.ijhm.2019.102387.

Romero, C., Mollá, A., Gómez-Borja, M.A. (2009): "Navigational web design and consumer behaviour: 'hierarchical tree' versus 'free network', Revista Europea de Direccion y Economia de la Empresa, vol. 18, nº 3, pp. 115-142.

Salén, H. (1987): Distribución y merchandising, Distribución Consulting, Madrid.

Sci ${ }^{2}$ s: Research Group “Soft Computing and Intelligent Information Systems” (2011): SciMAT (v. 1.0) [Software], University of Granada, Granada, disponible online en: http://sci2s.ugr.es/scimat/.

Talukdar, D. y Ariharan, V.G. (2011): "Empirical Regularity in Academic Research Productivity Patterns in Marketing”, International Journal of Research in Marketing, vol. 28, no 3, pp. 248-257.

Viedma-del-Jesus, M. I., Perakakis, P., Muñoz, M. A., Lopez-Herrera, A.G. y Vila, J. (2011): "Sketching the first forty-five years of the Journal Psychophysiology (1964-2008): A Co-word based analysis”, Psychophysiology, n 48, pp. 1029-1036.

Vogel, R. y Güttel, W.H. (2012): “The Dynamic Capability View in Strategic Management: A Bibliometric Review”, International Journal of Management Reviews, vol. 15, no 4, pp. 426-446. 
Wexter Box (2005): “El libre servicio en la distribución”, Wexter Box Marketing Consulting, disponible on line en: www.wexterbox.com.

Whittaker, J. (1989): “Creativity and conformity in science: Titles, keywords, and co-word analysis”, Social Science in Science, ${ }^{\circ}$ 19, pp. 473-96.

Widyastuti (2018): "Does visual merchandising, store atmosphere and private label product influence impulse buying? Evidence in Jakarta”, Journal of Business and Retail Management Research, vol. 12, n 3, pp. 140-148.

Willems, K., Brengman, M. y Van de Sanden, S. (2017): "In-store proximity marketing: experimenting with digital point-of-sales communication”, International Journal of Retail \& Distribution Management, vol. 45, $\mathrm{n}^{\circ}$ 7, pp, 910-927.

Zorrilla, P. (2002): "Nuevas tendencias en el merchandising. Generar experiencias para conquistar emociones y fidelizar clienes”, Distribución y Consumo, vol. 13, n 20, pp. 13-21.

Delgado, E. y Repiso, R. (2013): "The impact of scientific journals of communication: Comparing Google Scholar Metrics, Web of Science and Scopus”, Comunicar, vol. 21 $n^{\circ} 41$, pp. 45-52.

Vieira, E. S. y Gomes, J.A.N.F. (2009): “A comparison of Scopus and Web of Science for a typical university”, Scientometrics, 81 (November), pp. 587-604. 